\title{
Two-Higgs doublet solution to the LSND, MiniBooNE and muon $g-2$ anomalies
}

\author{
Waleed Abdallah $\odot,{ }^{1,2, *}$ Raj Gandhi $\oplus^{1, \dagger}$ and Samiran Roy $\oplus^{3, \$}$ \\ ${ }^{1}$ Harish-Chandra Research Institute, Chhatnag Road, Jhunsi, Allahabad 211019, India \\ ${ }^{2}$ Department of Mathematics, Faculty of Science, Cairo University, Giza 12613, Egypt \\ ${ }^{3}$ Physical Research Laboratory, Ahmedabad-380009, Gujarat, India
}

(Received 26 December 2020; accepted 21 July 2021; published 23 September 2021)

\begin{abstract}
We show that one of the simplest extensions of the Standard Model, the addition of a second Higgs doublet, when combined with a dark sector singlet scalar, allows us to $i$ ) explain the long-standing anomalies in the Liquid Scintillator Neutrino Detector (LSND) and MiniBooNE (MB) while maintaining compatibility with the null result from KARMEN, $i$ ) obtain, in the process, a portal to the dark sector, and $\mathrm{iii}$ ) comfortably account for the observed value of the muon $g-2$. Three singlet neutrinos allow for an understanding of observed neutrino mass-squared differences via a Type I seesaw, with two of the lighter states participating in the interaction in both LSND and MB. We obtain very good fits to energy and angular distributions in both experiments. We explain features of the solution presented here and discuss the constraints that our model must satisfy. We also mention prospects for future tests of its particle content.
\end{abstract}

DOI: 10.1103/PhysRevD.104.055028

\section{INTRODUCTION}

The rocklike stability of the Standard Model (SM) [1] has provided a powerfully reliable framework for both theoretical and experimental progress in particle physics over many decades. It cannot, however, be denied that over this period, the consistent agreement of experimental data (in particular from colliders) with its predictions has also been a source of frustration. This is especially so since there are undeniably strong qualitative reasons, coupled with physical evidence, to expect that there must be physics beyond the ambit of the SM. These reasons, and this evidence, include $a$ ) dark matter (DM) [2-6]; $b$ ) the observed matter and antimatter asymmetry in our Universe $[1,7,8] ; c)$ the existence of small but nonzero neutrino mass differences [9-12]; with masses widely different in magnitude from those of the charged leptons and quarks; and $d$ ) the existence, unsupported by compelling physical reasons, of three families of quarks and leptons with mixings and a large mass hierarchy.

\footnotetext{
awaleed@sci.cu.edu.eg

†raj@hri.res.in

¥samiran@prl.res.in
}

Published by the American Physical Society under the terms of the Creative Commons Attribution 4.0 International license. Further distribution of this work must maintain attribution to the author(s) and the published article's title, journal citation, and DOI. Funded by SCOAP .
Parallelly, albeit on a relatively smaller scale, extremely important experimental efforts in noncollider settings have supplemented and buttressed the search for new physics. It has gradually become evident that the landscape here is less bleak, and at present one can point to several experiments which report statistically significant discrepancies with respect to the predictions of the SM. Some anomalous results which have garnered attention and spurred significant activity in an effort to understand their origin are $a$ ) excesses in electron events in short-baseline neutrino experiments, which are now in tension with muon neutrino disappearance data [13] if interpreted as oscillation effects involving a sterile neutrino; $b$ ) observed discrepancies in the values of the anomalous magnetic moment of the muon [14] and the electron [15]; c) a significant excess in the signal versus background expectation in the KOTO experiment [16], which searches for the decay of a neutral kaon to a neutral pion and a neutrino pair; $d$ ) discrepancies with SM predictions in observables related to $B$ decays [17]; and finally, $e$ ) anomalies in the decay of excited states of beryllium [18].

Our focus in this work is on a subset of results in category a) above. Specifically, we address the Liquid Scintillator Neutrino Detector (LSND) excess (e.g., Ref. [19]) and the MiniBooNE (MB) low-energy excess (LEE) (e.g., Ref. [20]). In addition to having appreciable statistical significance, they have withstood scrutiny by both theoretical and experimental communities over a period of time. It is thus possible that these results in particular indicate genuine pointers to new physics, as opposed to un-understood backgrounds or 
detector-specific effects. ${ }^{1}$ The solution proposed here also helps resolve the discrepancy between the measured (see, e.g., Ref. [21]) and theoretically predicted (e.g., Ref. [22]) values of the anomalous magnetic moment of the muon.

We show that one of the simplest possible extensions of the SM, the addition of a second Higgs doublet, when acting as a portal to the dark sector, connects and provides an understanding of all three discrepant results mentioned above. Its function as a portal is achieved via its mixing with a dark (i.e., SM singlet) scalar. This mixing in the scalar sector allows heavier dark neutrinos coupled to the singlet scalar become part of the link between the SM and the dark sector. The dark neutrinos play two additional roles: $a$ ) they participate in the interaction that we use to explain the excess events in LSND and $\mathrm{MB}$, and $b$ ) they help generate neutrino masses via a seesaw mechanism. This lends synergy and economy to the model, the specifics of which we give below. It provides excellent fits to both energy and angular event distributions at LSND and MB.

Our paper is organized as follows: Section II briefly gives the specifics of the MB and LSND anomalies and has a brief discussion of the observed discrepancy in the value of the muon $g-2$. Section III describes $i$ ) the Lagrangian of our model and its particle content, $i i$ ) how the couplings of the additional scalars to fermions arise, and iii) the generation of neutrino masses. Section IV focuses on the interaction we use to explain the MB and LSND excesses. Section $\mathrm{V}$ gives our results and provides an accompanying discussion of their important features. Section VI discusses the constraints on our model. Section VII provides a concluding discussion, and indicates possible future tests of the model.

\section{THE MB, LSND, AND MUON $g-2$ ANOMALIES}

\section{A. Event excesses in MB and LSND}

Two low-energy neutrino experiments, MB (see Ref. [20] and references therein) and LSND (see Ref. [19], and references therein), have observed electronlike event excesses. Over time, it has become evident that the results of both cannot easily be explained within the ambit of the SM.

MB, based at Fermilab, uses muon neutrino and antineutrino beams produced by $8 \mathrm{GeV}$ protons impinging upon a beryllium target. The neutrino fluxes peak at around $600 \mathrm{MeV}\left(\nu_{\mu}\right)$ and around $400 \mathrm{MeV}\left(\bar{\nu}_{\mu}\right)$. The detector consists of a 40-foot-diameter sphere containing 818 tons of pure mineral oil $\left(\mathrm{CH}_{2}\right)$ and is located $541 \mathrm{~m}$ from the target. Starting in 2002, the MB experiment has up to 2019 collected a total of $11.27 \times 10^{20}$ protons on target (POT) in

\footnotetext{
${ }^{1}$ With regard to LSND and MB, which share many similarities in their overall physics goals and parameter reach (e.g., the ratio of oscillation length versus energy of the neutrino beam), we note that such attribution to their results requires two distinct "mundane" explanations, given that they differ very significantly in backgrounds and systematic errors.
}

antineutrino mode and $18.75 \times 10^{20} \mathrm{POT}$ in neutrino mode. Electronlike event excesses of $560.6 \pm 119.6$ in the neutrino mode, and $79.3 \pm 28.6$ in the antineutrino mode, with an overall significance of $4.8 \sigma$ have been established in the neutrino energy range $200 \mathrm{MeV}<E_{\nu}^{Q E}<1250 \mathrm{MeV}$. Most of the excess is confined to the range $100 \mathrm{MeV}<$ $\mathrm{E}_{\mathrm{vis}}<700 \mathrm{MeV}$ in visible energy, with a somewhat forward angular distribution, and is referred to as the MB LEE. We note $a$ ) that all major backgrounds are constrained by in situ measurements, and $b$ ) that $\mathrm{MB}$, due to being a mineral oil Cerenkov light detector, cannot distinguish photons from electrons in the final state. Additionally, under certain conditions, MB could also misidentify an $e^{+} e^{-}$pair as a single electron or positron.

LSND was a detector with 167 tons of mineral oil, doped with scintillator. It employed neutrino and antineutrino beams originating from $\pi^{-}$DIF as well as $\mu$ decay at rest (DAR). The principal detection interaction was the inverse beta decay process, $\bar{\nu}_{e}+p \rightarrow e^{+}+n$. The detector looked for Cherenkov and scintillation light associated with the $e^{+}$ and the correlated and delayed scintillation light from the neutron capture on hydrogen, producing a $2.2 \mathrm{MeV} \gamma$. The experiment observed $87.9 \pm 22.4 \pm 6.0$ such events above expectations at a significance of $3.8 \sigma$, over its run span from 1993 to 1998 at the Los Alamos Accelerator National Laboratory. For reasons similar to those at MB, LSND lacked the capability to discriminate a photon signal from those of an $e^{+}, e^{-}$, or an $e^{+} e^{-}$pair.

In addition, we mention the KARMEN experiment [23], which, like LSND and MB, employed a mineral oil detection medium, but was less than a third of the size of LSND. It had similar incoming proton energy and efficiencies. Unlike LSND, it saw no evidence of an excess.

There have been numerous attempts to understand both of these excesses. A widely discussed resolution involves the presence of sterile neutrinos with mass-squared values of $\sim 1-10 \mathrm{eV}^{2}$, oscillating to SM neutrinos, leading to $\bar{\nu}_{e}$ and $\nu_{e}$ appearance [24]. It is partially supported by deficits in $\nu_{e}$ events in radioactive source experiments and in $\bar{\nu}_{e}$ reactor flux measurements, as well as results from the reactor experiments. However, this explanation for LSND and $\mathrm{MB}$ excesses has had to contend with gradually increasing tension with disappearance experiments and is also disfavored by cosmological data. For recent global analyses, a full set of references, and more detailed discussions of these issues, the reader is referred to Refs. [25-31].

The tightening of constraints and parameter space for the sterile-active hypothesis has, in turn, led to a large number of proposals to explain one or both of the LSND and MB excesses via new physics [32-49]. Many of these scenarios also face a significant number of constraints. For a discussion of these and for related references, we refer the reader to Refs. [50-53]. It is, however, fair to say that at the present time, the search for a compelling and simultaneous 
explanation of both the LSND and MB anomalies remains a challenge [54].

\section{B. The muon $g-2$ anomaly}

The Lande $g$ factor, and its deviation from the tree-level value of 2 , represents one of the most precisely measured quantities in the SM. It thus is also an excellent probe for new physics. Currently there exists a long-standing and statistically significant discrepancy between its measurement [21,55] and the theoretically predicted value, which involves contributions from quantum electrodynamics, quantum chromodynamics, and electroweak theory $[14,22,56,57]$. Specifically,

$$
\Delta a_{\mu}=a_{\mu}^{\text {meas }}-a_{\mu}^{\text {theory }}=(2.74 \pm 0.73) \times 10^{-9} .
$$

There have been many proposals for new physics which provide possible explanations for this discrepancy (for reviews and a full list of references, see Refs. [14,22, $56,57]$.). Our attempt in this work, details of which are provided in the sections to follow, is related to a class of possible solutions suggested by several authors [58-69] involving a light scalar with a mass in the sub-GeV range and a relatively weak coupling to muons.

\section{THE MODEL}

We extend the scalar sector of the SM by incorporating a second Higgs doublet-i.e., the widely studied two-Higgsdoublet model (2HDM) [70,71] in addition to a dark singlet real scalar ${ }^{2} \phi_{h^{\prime}}$. In addition, three right-handed neutrinos help generate neutrino masses via the seesaw mechanism and participate in the interaction described in the next section.

We write the scalar potential $V$ in the Higgs basis $\left(\phi_{h}, \phi_{H}, \phi_{h^{\prime}}\right)$ [72,73], with $\lambda_{i}$ denoting the usual set of quartic couplings:

$$
\begin{aligned}
V & =\left|\phi_{h}\right|^{2}\left(\frac{\lambda_{1}}{2}\left|\phi_{h}\right|^{2}+\lambda_{3}\left|\phi_{H}\right|^{2}+\mu_{1}\right) \\
& +\left|\phi_{H}\right|^{2}\left(\frac{\lambda_{2}}{2}\left|\phi_{H}\right|^{2}+\mu_{2}\right)+\lambda_{4}\left(\phi_{h}^{\dagger} \phi_{H}\right)\left(\phi_{H}^{\dagger} \phi_{h}\right) \\
& +\phi_{h^{\prime}}^{2}\left(\lambda_{2}^{\prime} \phi_{h^{\prime}}^{2}+\lambda_{3}^{\prime}\left|\phi_{h}\right|^{2}+\lambda_{4}^{\prime}\left|\phi_{H}\right|^{2}+m^{\prime} \phi_{h^{\prime}}+\mu^{\prime}\right) \\
& +\left[\phi_{h}^{\dagger} \phi_{H}\left(\frac{\lambda_{5}}{2} \phi_{h}^{\dagger} \phi_{H}+\lambda_{6}\left|\phi_{h}\right|^{2}+\lambda_{7}\left|\phi_{H}\right|^{2}+\lambda_{5}^{\prime} \phi_{h^{\prime}}^{2}-\mu_{12}\right)\right. \\
& \left.+\phi_{h^{\prime}}\left(m_{1}\left|\phi_{h}\right|^{2}+m_{2}\left|\phi_{H}\right|^{2}+m_{12} \phi_{h}^{\dagger} \phi_{H}\right)+\text { H.c. }\right],
\end{aligned}
$$

\footnotetext{
${ }^{2}$ The introduction of scalars in order to explain one or more of the anomalies at noncollider experiments mentioned in the Introduction is a feature of many recent papers-e.g., Refs. [4448,58-69]. Our model resembles the approach taken in Refs. $[47,48]$. In particular, it is essentially a more economical version of the model in Ref. [48], without an additional $U(1)$.
}

where

$$
\begin{gathered}
\phi_{h}=\left(\begin{array}{c}
H_{1}^{+} \\
\frac{v+H_{1}^{0}+i G^{0}}{\sqrt{2}}
\end{array}\right) \equiv \cos \beta \Phi_{1}+\sin \beta \Phi_{2}, \\
\phi_{H}=\left(\begin{array}{c}
H_{2}^{+} \\
\frac{H_{2}^{0}+i A^{0}}{\sqrt{2}}
\end{array}\right) \equiv-\sin \beta \Phi_{1}+\cos \beta \Phi_{2}, \\
\phi_{h^{\prime}}=H_{3}^{0} / \sqrt{2},
\end{gathered}
$$

so that $v^{2}=v_{1}^{2}+v_{2}^{2} \simeq(246 \mathrm{GeV})^{2}$ and $\tan \beta=v_{2} / v_{1}$, where $\left\langle\Phi_{i}\right\rangle=v_{i} / \sqrt{2},\left\langle\phi_{h}\right\rangle=v$, while $\left\langle\phi_{H}\right\rangle=0=\left\langle\phi_{h^{\prime}}\right\rangle$ and $v, v_{i}$ denote vacuum expectation values (VEVs). Here, $|\phi|^{2} \equiv \phi^{\dagger} \phi$ and $H_{1}^{+}, G^{0}$ are the Goldstone bosons, which give the gauge bosons mass after the electroweak symmetry is spontaneously broken. The mass matrix of the neutral $C P$-even Higgses in the basis $\left(H_{1}^{0}, H_{2}^{0}, H_{3}^{0}\right)$ is given by

$$
m_{\mathcal{H}}^{2}=\left(\begin{array}{ccc}
\lambda_{1} v^{2} & \lambda_{6} v^{2} & 0 \\
\lambda_{6} v^{2} & \mu_{H} & m_{12} v / \sqrt{2} \\
0 & m_{12} v / \sqrt{2} & \mu_{h^{\prime}}
\end{array}\right),
$$

where $\mu_{H}=\mu_{2}+\left(\lambda_{3}+\lambda_{4}+\lambda_{5}\right) v^{2} / 2$ and $\mu_{h^{\prime}}=\mu^{\prime}+$ $\lambda_{3}^{\prime} v^{2} / 2$. Here, we have used the following minimization conditions of the scalar potential $V$ :

$$
\mu_{1}=-\frac{1}{2} \lambda_{1} v^{2}, \quad \mu_{12}=\frac{1}{2} \lambda_{6} v^{2}, \quad m_{1}=0 .
$$

The matrix in Eq. (6), $m_{\mathcal{H}}^{2}$, is diagonalized by $Z^{\mathcal{H}}$ as follows:

$$
\begin{gathered}
Z^{\mathcal{H}} m_{\mathcal{H}}^{2}\left(Z^{\mathcal{H}}\right)^{T}=\left(m_{\mathcal{H}}^{2}\right)^{\text {diag }}, \quad \text { with } \quad H_{i}^{0}=\sum_{j} Z_{j i}^{\mathcal{H}} h_{j}, \\
Z^{\mathcal{H}}=\left(\begin{array}{ccc}
1 & 0 & 0 \\
0 & c_{\delta} & -s_{\delta} \\
0 & s_{\delta} & c_{\delta}
\end{array}\right), \quad \tan 2 \delta=\frac{\sqrt{2} m_{12} v}{\mu_{h^{\prime}}-\mu_{H}},
\end{gathered}
$$

where $i) s_{\delta} \equiv \sin \delta, c_{\delta} \equiv \cos \delta,\left(h_{1}, h_{2}, h_{3}\right)=\left(h, H, h^{\prime}\right)$ are the mass eigenstates; ii) $H_{1}^{0} \approx h$ is the SM-like Higgs in the alignment limit (i.e., $\lambda_{6} \sim 0$ ) assumed here; and iii) $m_{h}^{2} \simeq \lambda_{1} v^{2}$. The masses of the extra $C P$-even physical Higgs states $\left(H, h^{\prime}\right)$ are given by

$$
m_{H, h^{\prime}}^{2} \simeq \frac{1}{2}\left[\mu_{H}+\mu_{h^{\prime}} \pm \sqrt{\left(\mu_{H}-\mu_{h^{\prime}}\right)^{2}+2 m_{12}^{2} v^{2}}\right] .
$$

Also, the charged and $C P$-odd Higgs masses, respectively, are given by

$$
m_{H^{ \pm}}^{2}=\mu_{2}+\lambda_{3} v^{2} / 2
$$




$$
m_{A}^{2}=\mu_{2}+\left(\lambda_{3}+\lambda_{4}-\lambda_{5}\right) v^{2} / 2 .
$$

In the Higgs basis, the relevant Lagrangian $\mathcal{L}$ can be written as follows:

$$
\begin{aligned}
\mathcal{L}= & \sqrt{2}\left[\left(X_{i j}^{u} \tilde{\phi}_{h}+\bar{X}_{i j}^{u} \tilde{\phi}_{H}\right) \bar{Q}_{L}^{i} u_{R}^{j}+\left(X_{i j}^{d} \phi_{h}+\bar{X}_{i j}^{d} \phi_{H}\right) \bar{Q}_{L}^{i} d_{R}^{j}\right. \\
& +\left(X_{i j}^{e} \phi_{h}+\bar{X}_{i j}^{e} \phi_{H}\right) \bar{L}_{L}^{i} e_{R}^{j}+\left(X_{i j}^{\nu} \tilde{\phi}_{h}+\bar{X}_{i j}^{\nu} \tilde{\phi}_{H}\right) \bar{L}_{L}^{i} \nu_{R_{j}} \\
& \left.+\frac{1}{\sqrt{8}} m_{i j} \bar{\nu}_{R_{i}}^{c} \nu_{R_{j}}+\lambda_{i j}^{N} \bar{\nu}_{R_{i}}^{c} \phi_{h^{\prime}} \nu_{R_{j}}+\text { H.c. }\right]
\end{aligned}
$$

where

$$
X_{i j}^{k}=Y_{i j}^{k} c_{\beta}+\tilde{Y}_{i j}^{k} s_{\beta}, \quad \bar{X}_{i j}^{k}=-Y_{i j}^{k} s_{\beta}+\tilde{Y}_{i j}^{k} c_{\beta},
$$

and $Y^{k}, \tilde{Y}^{k}$ are the Yukawa couplings in the $\left(\Phi_{1}, \Phi_{2}\right)$ basis. We note that $X_{i j}^{k}$ and $\bar{X}_{i j}^{k}$ are independent Yukawa matrices. The fermion masses receive contributions only from $X_{i j}^{k}$, since in the Higgs basis only $\phi_{h}$ acquires a nonzero VEV while $\left\langle\phi_{H}\right\rangle=0=\left\langle\phi_{h^{\prime}}\right\rangle$, leading to $X^{k}=\mathcal{M}_{k} / v$, where $\mathcal{M}_{k}$ are the fermion mass matrices. In this basis, $\bar{X}_{i j}^{k}$ are free parameters and nondiagonal matrices. Hereafter, we work in a basis in which the fermion (leptons and quarks) mass matrices are real and diagonal, where $U_{k} \mathcal{M}_{k} V_{k}^{\dagger}=m_{k}^{\text {diag }}$ are their bi-unitary transformations.

After rotation, one finds the following coupling strengths of the scalars $h, h^{\prime}$, and $H$ with fermions (leptons and quarks), respectively:

$y_{f}^{h}=\frac{m_{f}}{v}, \quad y_{f}^{h^{\prime}}=y^{f} Z_{32}^{\mathcal{H}}=y^{f} s_{\delta}, \quad y_{f}^{H}=y^{f} Z_{22}^{\mathcal{H}}=y^{f} c_{\delta}$,

where $m_{f}$ are the SM fermion masses and $y^{f}$ are the diagonal elements of the rotated $\bar{X}^{f}$ which are independent of the Yukawa couplings $\left(y_{f}^{h}=m_{f} / v\right)$ of the SM Higgsfermions interactions. $\delta$ manifestly becomes the scalar mixing angle between the mass eigenstates $\left(H, h^{\prime}\right)$ and the gauge eigenstates $\left(H_{2}^{0}, H_{3}^{0}\right)$. For neutrinos, we define $n_{R_{i}}=\left(U_{\nu_{R}}\right)_{i j} \nu_{R_{j}}$ and $n_{L_{i}}=\left(U_{\nu_{L}}\right)_{i j} \nu_{L_{j}}$ such that the matrices $\mathcal{M}_{\nu}\left(=v X^{\nu}\right)$ and $m$ can be diagonalized as follows:

$$
U_{\nu_{L}} \mathcal{M}_{\nu} U_{\nu_{R}}^{\dagger}=m_{D}^{\text {diag }}, \quad U_{\nu_{R}} m U_{\nu_{R}}^{\dagger}=m_{\nu_{R}}^{\text {diag }} .
$$

One can then define the following matrices:

$$
\lambda^{n}=U_{\nu_{R}} \lambda^{N} U_{\nu_{R}}^{\dagger}, \quad y^{\nu}=U_{\nu_{L}} \bar{X}^{\nu} U_{\nu_{R}}^{\dagger} .
$$

The part of the Lagrangian describing neutrino masses and interactions is then given by

$$
\begin{aligned}
\mathcal{L}_{\nu}= & m_{D_{i}}^{\mathrm{diag}} \bar{n}_{L_{i}} n_{R_{i}}+\frac{1}{2} m_{\nu_{R_{i}}}^{\mathrm{diag}} \bar{n}_{R_{i}}^{c} n_{R_{i}}+y_{\nu_{i j}}^{\phi} \bar{n}_{L_{i}} n_{R_{j}} \phi \\
& +\left(\lambda_{N_{i j}}^{h^{\prime}} h^{\prime}+\lambda_{N_{i j}}^{H} H\right) \bar{n}_{R_{i}}^{c} n_{R_{j}}+\text { H.c. },
\end{aligned}
$$

where $\phi=A, h, H, h^{\prime}$.

Consequently, the neutrino mass Lagrangian becomes

$$
\mathcal{L}_{\nu}^{m}=\frac{1}{2}\left(\bar{n}_{L}^{c} \bar{n}_{R}\right)\left(\begin{array}{cc}
0 & m_{D}^{\text {diag }} \\
m_{D}^{\text {diag }} & m_{\nu_{R}}^{\text {diag }}
\end{array}\right)\left(\begin{array}{c}
n_{L} \\
n_{R}^{c}
\end{array}\right)+\text { H.c. },
$$

and the neutrino mass matrix is given by

$$
m_{\nu}=\left(\begin{array}{cc}
0 & m_{D}^{\text {diag }} \\
m_{D}^{\text {diag }} & m_{\nu_{R}}^{\text {diag }}
\end{array}\right) \rightarrow m_{\nu}^{\text {diag }}=\mathcal{N} m_{\nu} \mathcal{N}^{\dagger} .
$$

Its eigenvalues $\left(m_{\nu}^{\text {diag }}\right)$ are

$m_{\nu}^{\text {diag }} \simeq \operatorname{diag}\left\{-m_{D_{i}}^{2} / m_{\nu_{R_{i}}}, m_{\nu_{R_{i}}}\right\}, \quad(i=1,2,3)$.

The neutrino mass matrix $m_{\nu}$ can be diagonalized, up to $\mathcal{O}\left(m_{D_{i}} / m_{\nu_{R_{i}}}\right)$, by the neutrino mixing matrix $\mathcal{N}$, which can be written, up to corrections of $\mathcal{O}\left(m_{D_{i}}^{2} / m_{\nu_{R_{i}}}^{2}\right)$, as

$$
\mathcal{N} \simeq\left(\begin{array}{cc}
I-\Theta^{2} / 2 & \Theta \\
-\Theta & I-\Theta^{2} / 2
\end{array}\right)
$$

where $\Theta_{i}=m_{D_{i}} / m_{\nu_{R_{i}}}$. The neutrino mass eigenstates (physical states) are given by

$$
\left(\begin{array}{c}
\nu \\
N
\end{array}\right)=\left(\begin{array}{cc}
I-\Theta^{2} / 2 & \Theta \\
-\Theta & I-\Theta^{2} / 2
\end{array}\right)\left(\begin{array}{l}
n_{L} \\
n_{R}^{c}
\end{array}\right) .
$$

For the normal order $\left(m_{\nu_{1}}<m_{\nu_{2}}<m_{\nu_{3}}\right)$, the two masssquared differences of the light neutrinos determined from the oscillation data are $\Delta m_{21}^{2}=(7.05-8.14) \times 10^{-5} \mathrm{eV}^{2}$ and $\Delta m_{31}^{2}=(2.41-2.60) \times 10^{-3} \mathrm{eV}^{2}$ [74]. We have chosen a benchmark point (see Table I) so that it satisfies these values. Finally, the part of the Lagrangian specifying neutrino interactions is given by

$$
\mathcal{L}_{\nu}^{\text {int }} \simeq y_{\nu_{i j}}^{\phi} \bar{\nu}_{i} N_{j} \phi+\left(\lambda_{N_{i j}}^{h^{\prime}} h^{\prime}+\lambda_{N_{i j}}^{H} H\right) \bar{N}_{i} N_{j}+\text { H.c. },
$$

where the coupling strengths of the scalars $h^{\prime}, H$ for vertices connecting active and sterile neutrinos, respectively, are as follows:

$$
y_{\nu_{i j}}^{h^{\prime}}=y_{i j}^{\nu} Z_{32}^{\mathcal{H}}=y_{i j}^{\nu} s_{\delta}, \quad y_{\nu_{i j}}^{H}=y_{i j}^{\nu} Z_{22}^{\mathcal{H}}=y_{i j}^{\nu} c_{\delta} .
$$

Additionally, the coupling strengths of the scalars $h^{\prime}, H$ for vertices connecting two sterile states, respectively, are 
TABLE I. Benchmark point used for event generation in LSND, MB and for calculating the muon $g-2$.

\begin{tabular}{lccccc}
\hline \hline$m_{N_{1}}$ & $m_{N_{2}}$ & $m_{N_{3}}$ & $y_{u}^{h^{\prime}(H)} \times 10^{6}$ & $y_{e(\mu)}^{h^{\prime}} \times 10^{4}$ & $y_{e(\mu)}^{H} \times 10^{4}$ \\
\hline $85 \mathrm{MeV}$ & $130 \mathrm{MeV}$ & $10 \mathrm{GeV}$ & $0.8(8)$ & $0.23(1.6)$ & $2.29(15.9)$ \\
\hline \hline$m_{h^{\prime}}$ & $m_{H}$ & $\sin \delta$ & $y_{d}^{h^{\prime}(H)} \times 10^{6}$ & $y_{\nu_{i 2}}^{h^{\prime}(H)} \times 10^{3}$ & $\lambda_{N_{12}}^{h^{\prime}(H)} \times 10^{3}$ \\
\hline $17 \mathrm{MeV}$ & $750 \mathrm{MeV}$ & 0.1 & $0.8(8)$ & $1.25(12.4)$ & $74.6(-7.5)$ \\
\hline \hline
\end{tabular}

$\lambda_{N_{i j}}^{h^{\prime}}=\lambda_{i j}^{n} Z_{32}^{\mathcal{H}}=\lambda_{i j}^{n} c_{\delta}, \quad \lambda_{N_{i j}}^{H}=\lambda_{i j}^{n} Z_{22}^{\mathcal{H}}=-\lambda_{i j}^{n} s_{\delta}$.

Finally, we stress that all the Yukawa couplings of the light scalars $\left(h^{\prime}, H\right)$-fermion interactions $\left(y_{f}^{h^{\prime}, H}\right.$, $\left.f=\ell, q, \nu_{\ell}, N\right)$ are free and independent of the Yukawa couplings $\left(y_{f}^{h}=m_{f} / v\right)$ of the SM Higgs-fermion interactions.

\section{THE INTERACTION IN MB AND LSND}

In the process shown in Fig. 1, the heavy sterile neutrino $N_{2}$ is produced via the upscattering of a muon neutrino $\left(\nu_{\mu}=U_{\mu i} \nu_{i}\right)$ present in the beam, both for MB and LSND. Once $N_{2}$ is produced, it decays promptly to another lighter sterile neutrino $N_{1}$ and a light scalar $h^{\prime}$. In our scenario, $N_{1}$ is a long-lived particle that either escapes the detector or decays to lighter dark particles, but $h^{\prime}$ decays promptly to a collimated $e^{+} e^{-}$pair and produces the visible light that comprises the signal.

As shown in Fig. 1, both $H$ and $h^{\prime}$ act as mediators and contribute to the total cross section. The contribution of $h^{\prime}$ is much smaller $(\sim 10 \%)$ compared to that of $H$, since $\sin \delta \simeq 0.1$. However, this small contribution plays an important role in producing the correct angular distribution in $\mathrm{MB}$, as we discuss later. In our model, $H$ and $h^{\prime}$ predominantly couple to the first generation of quarks ( $u$ and $d$ ) and have negligible or tiny couplings to other families. The effective coupling $\left(F_{N}\right)$ of either scalar to a nucleon $(N)$ can be written as [75-77]

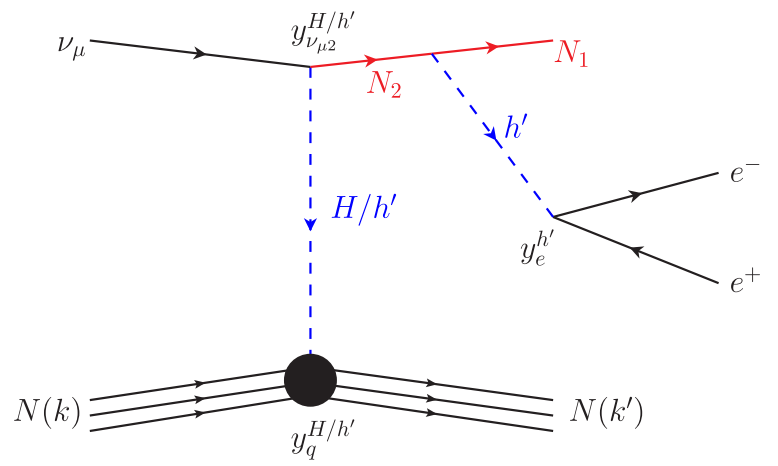

FIG. 1. Feynman diagram of the scattering process in our model which leads to the excess at LSND and MB.

$$
\frac{F_{N}}{M_{N}}=\sum_{q=u, d} f_{T_{q}}^{N} \frac{f_{q}}{m_{q}}
$$

Here $M_{N}$ is the nucleon mass, and the values of $\quad\left(f_{T_{u}}^{p}, f_{T_{d}}^{p}, f_{T_{u}}^{n}, f_{T_{d}}^{n}\right)=(0.020,0.041,0.0189,0.0451)$. In our scenario, $f_{q}=y_{q}^{H, h^{\prime}},(q=u, d)$.

We include both the incoherent and coherent contribution in the production of $N_{2}$ in MB. For LSND, however, we consider only incoherent scattering from neutrons. The total differential cross section, for the target in $\mathrm{MB}$-i.e., $\mathrm{CH}_{2}$-is given by 9.5

$$
\left[\frac{d \sigma}{d E_{N_{2}}}\right]_{\mathrm{CH}_{2}}=[\underbrace{\left(8 F_{p}+6 F_{n}\right)}_{\text {incoherent }}+\underbrace{\left.\left(6 F_{p}+6 F_{n}\right)^{2} e^{-2 b \mid q^{2}}\right]}_{\text {coherent }} \frac{d \sigma}{d E_{N_{2}}} .
$$

The entire carbon nucleus $\left(\mathrm{C}^{12}\right)$ contributes in coherent scattering, with, however, decreasing contributions as $\left|q^{2}\right|=\left|\left(k^{\prime}-k\right)^{2}\right|$ increases. This is implemented by the form factor $\exp \left(-2 b\left|q^{2}\right|\right)$ [78], where $b$ is a numerical parameter, which for $\mathrm{C}^{12}$ has been chosen to be $25 \mathrm{GeV}^{-2}$ $[78,79]$. The number of events is given by

$\mathrm{N}_{\text {events }}=\eta \int d E_{\nu} d E_{N_{2}} \frac{d \Phi^{\nu}}{d E_{\nu}} \frac{d \sigma}{d E_{N_{2}}} \times \operatorname{BR}\left(N_{2} \rightarrow N_{1} h^{\prime}\right)$,

with $E_{h^{\prime}} \in\left[E_{h^{\prime}}, E_{h^{\prime}}+\Delta E_{h^{\prime}}\right]$, and $\Phi^{\nu}$ is the incoming muon neutrino flux. $\eta$ contains all detector-related information like efficiencies, POT, etc. All calculations for LSND, MB and the value of the muon $g-2$ are carried out using the benchmark values in Table I. Finally, for these values, the calculated lifetimes of $N_{2}$ and $h^{\prime}$ in the rest frame are $10^{-17} \mathrm{~s}$ and $1.8 \times 10^{-12} \mathrm{~s}$, respectively.

Our results are presented in the next section.

\section{RESULTS AND DISCUSSION}

In this section, we present the results of our numerical calculations, using the cross section for the process and the model described in Sec. III.

\section{A. Results and discussion for MB and LSND}

Figure 2 (top panels) shows the MB data points, SM backgrounds, and the prediction of our model (blue solid 

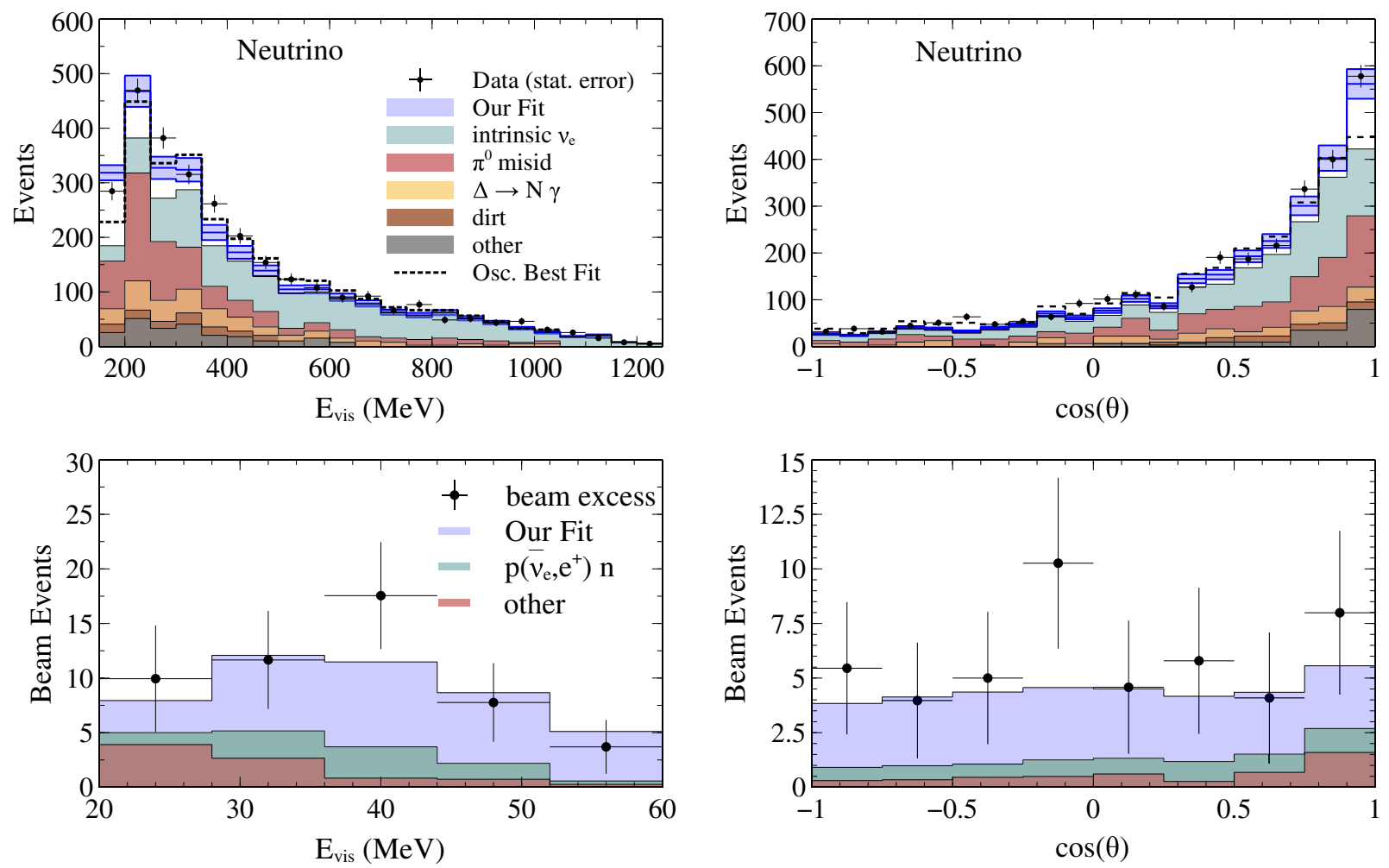

FIG. 2. Top panels: The MB electronlike events (backgrounds and signal) from Ref. [20], versus the visible energy $\mathrm{E}_{\mathrm{vis}}$ (left panel) and versus the cosine of the emitted angle of the light (right panel), for neutrino runs. The blue solid line is the prediction of our model. Bottom panels: The energy distribution (left panel) of the LSND data [19] for $R_{\gamma}>10$, and the angular distribution (right panel) of the light due to the electronlike final state, for $R_{\gamma}>1$. The shaded blue region in both panels is our fit, and other shaded regions are the backgrounds.

line) in each bin. Also shown (black dashed line) is the oscillation best fit. The latest data set for the neutrino mode, corresponding to $18.75 \times 10^{20} \mathrm{POT}$, as detailed in Ref. [20], has been used in our fit. The left panel shows the distribution of the measured visible energy, $\mathrm{E}_{\mathrm{vis}}$, plotted against the events for neutrinos. For our model, $\mathrm{E}_{\mathrm{vis}}$ corresponds to $E_{h^{\prime}}$. The right panel shows the corresponding angular distributions for the emitted light. The benchmark parameter values used to obtain the fit from our model are shown in Table I. The plots have been prepared using fluxes, efficiencies, POT exposures, and other relevant information from Ref. [80] and references therein. We see that very good fits to the data are obtained for both the energy and the angular distributions. The data points show only statistical uncertainties. We have assumed a $15 \%$ systematic uncertainty for our calculations. These errors are represented by the blue bands in the figures.

As mentioned earlier, the LSND observations measure the visible energy from the Cerenkov and scintillation light of an assumed electronlike event, as well as the $2.2 \mathrm{MeV}$ photon resulting from coincident neutron capture on hydrogen. In our model, this corresponds to the scattering diagrams in Fig. 1 where the target is a neutron in the carbon nucleus. Unlike the case of MB above, where both coherent and incoherent processes contribute to the total cross section, the LSND cross section we have used includes only an incoherent contribution. All necessary information on fluxes, efficiencies, POT, etc., for LSND has been taken from Ref. [19] and references therein.

Figure 2 (bottom-left panel) shows our results in comparison to the LSND data for $R_{\gamma}>10$, where $R_{\gamma}$ is a parameter defined by the LSND Collaboration (see, for instance, Ref. [19]) that represents a likelihood ratio that the observed photon signaling the presence of the neutron was correlated as opposed to being accidental. This plot shows the energy distribution and the excess events in the data, as well as those resulting from our model using the same benchmark parameters as were used to generate the MB results. We find a total of 28.7 events from our model, compared to the 32 events seen by LSND for this choice of $R_{\gamma}$.

Figure 2 (bottom-right panel) shows the angular distribution of the light due to the electronlike final state, for $R_{\gamma}>1$ and visible energies in the range $36 \mathrm{MeV}<$ $\mathrm{E}_{\mathrm{vis}}<60 \mathrm{MeV}$. In both panels, the blue shaded region

\footnotetext{
${ }^{3}$ The bottom panels use different ranges of $R_{\gamma}$ and $\mathrm{E}_{\mathrm{vis}}$, because we have chosen to present our results to correspond to the generally available results presented by the LSND Collaboration, which use different $R_{\gamma}$ and $\mathrm{E}_{\mathrm{vis}}$ ranges for the energy and angular distributions.
} 
is the result of our model, shown along with backgrounds and data.

Several points are pertinent to understanding the results obtained. We discuss them below:

(1) All LSND events in our scenario stem from the highenergy part of their DIF flux, which is kinematically capable of producing the $N_{2}\left(m_{N_{2}} \simeq 130 \mathrm{MeV}\right)$. This flux originates in $\pi^{+}$'s created in proton collisions in the LSND target (the experiment used two different targets over the running period-i.e., water and a high- $Z$ material). This leads to a beam of $\nu_{\mu}$ 's, which interacts in the detector via $\nu_{\mu} \mathrm{CH}_{2} \rightarrow$ $n N_{2} X \rightarrow n N_{1} h^{\prime} X \rightarrow N_{1} \gamma e^{+} e^{-} X$ (see Fig. 1). In the final step, the photon is the correlated $\gamma$ with an energy of $2.2 \mathrm{MeV}$, signifying the capture of the neutron by a nucleus. The decays of both $h^{\prime}$ and $N_{2}$ are prompt, while $N_{1}$ is either long-lived and escapes the detector or decays to lighter invisible states.

(2) In our scenario, both $H$ and $h^{\prime}$ act as mediators and contribute to the total cross section. The contribution of $h^{\prime}$ is much smaller $(\sim 10 \%)$ than that of $H$, since $\sin \delta \simeq 0.1$. However, this plays an important role in producing the correct angular distribution in MB. In particular, $h^{\prime}$ is responsible for a coherent contribution which helps sufficiently populate the first (i.e., most forward) bin in the top-right panel of Fig. 2.

(3) As a consequence of the heavy particle production $\left(N_{2}\right)$ necessary, our model would not give any signal in KARMEN, which has a narrow-band DIF flux that peaks at $\sim 30 \mathrm{MeV}$, hence making it compatible with their null result.

(4) The DIF flux, in the oscillation hypothesis, generates electronlike events in energy bins beyond $60 \mathrm{MeV}$. Indeed, LSND saw $10.5 \pm 4.9$ such events (without a correlated neutron) in the range $60 \mathrm{MeV}<\mathrm{E}_{\mathrm{vis}}<200 \mathrm{MeV}$, attributable to an oscillation probability of $(2.9 \pm 1.4) \times 10^{-3}$ [81]. Our model predicts 34 such events, which is within their acceptable range of uncertainty.

(5) LSND saw about 6 events with a correlated neutron in the energy range $60 \mathrm{MeV}<\mathrm{E}_{\mathrm{vis}}<200 \mathrm{MeV}$, and our calculations yield 5.6 such events, in agreement with their observations.

(6) As mentioned earlier, only incoherent neutron scattering contributes to the event counts in LSND. We have assumed $8 \mathrm{MeV}$ as the minimum energy transferred to a neutron in order to knock it out and register an event. Additionally, the masses of $\mathrm{N}_{2}$ and $N_{1}$ are important factors in obtaining both the correct number and the correct distributions in this detector. Lowering the mass of $N_{2}$ increases the total events significantly, since it provides access to lower energies in the DIF flux spectrum. Decreasing the mass of $N_{1}$ shifts the event peak towards higher visible energies, and leads to higher numbers of correlated neutron events with energies $>60 \mathrm{MeV}$, which would conflict with what LSND saw. On the other hand, in $\mathrm{MB}$, the effects of $N_{2}$ and $N_{1}$ masses do not play as significant a role as they do in LSND, although the MB energy distribution improves if the $N_{1}$ mass is decreased from our current benchmark value.

(7) Finally, we note that the criteria as to when an $e^{+} e^{-}$ pair constitutes a signal that may be counted as an electronlike event in both detectors are different. $\mathrm{MB}$ is not able to distinguish an $e^{+} e^{-}$pair from a single electron [52,82] if the invariant mass of $e^{+} e^{-}<30 \mathrm{MeV}$, or if the angle between the pair is $5^{\circ}$ or less. In our scenario, the mass of $h^{\prime}$, and hence that of the pair, is $17 \mathrm{MeV}$.

In LSND, the visible energies are quite low compared to those in MB. Hence, the opening angle of the $e^{+} e^{-}$pair can be large for the lower end of the visible energy ( 20-30 MeV). However, LSND did not attempt to search for $e^{+} e^{-}$pairs or $\gamma \gamma$ pairs, and for this reason it is reasonable to assume that it would reconstruct most $e^{+} e^{-}$pairs as a singleelectron event. In particular, because timing was their most powerful particle-identifying variable, the fit to a Cherenkov ring would select the most significant ring, even for large angles between the $e^{+} e^{-}$pair. Therefore, $e^{+} e^{-}$pairs with correlated neutrons would explain the LSND excess [83], especially since no known $e^{+} e^{-}$or $\gamma \gamma$ backgrounds were expected in LSND. A more accurate calculation than the simple one performed here would incorporate the effects of fitting only the most energetic ring out of two which have a large angle between them. One effect of this would be to slightly increase the events in the middle bin $(36-44 \mathrm{MeV})$ at the expense of those at higher energies (including those with energy $>60 \mathrm{MeV}$ ). It is evident from Fig. 2 (bottom-left panel) that this would improve the fit shown.

\section{B. Muon anomalous magnetic moment}

The one-loop contribution of a scalar $\phi$ to the muon anomalous magnetic dipole moment is given by $[84,85]$

$$
\Delta a_{\mu}=\frac{\left(y_{\mu}^{\phi}\right)^{2}}{8 \pi^{2}} \int_{0}^{1} d x \frac{(1-x)^{2}(1+x)}{(1-x)^{2}+x r_{\phi}^{2}}
$$

where $r_{\phi}=m_{\phi} / m_{\mu}$, and $\phi=h^{\prime}, H . y_{\mu}^{\phi}$ is the coupling strength of the scalar $\phi$ with the $\mu^{+} \mu^{-}$pair, which is defined in Eq. (15).

We note that first, $m_{h^{\prime}}, m_{H}$ are fixed to fit the LSND and MB measurements. Also, both $h^{\prime}$ and $H$ contribute to the total muon anomalous magnetic moment $\Delta a_{\mu}$. Their ratio $\Delta a_{\mu}^{h^{\prime}} / \Delta a_{\mu}^{H} \sim \tan ^{2} \delta$. In a more general situation, $y^{\mu}$ and the 
angle $\delta$ would correspond to free parameters, and one can fix them to fit the central value for $\Delta a_{\mu}$.

For a suitably selected combination of $y^{\mu}$ and $\delta\left(y^{\mu}=\right.$ $1.6 \times 10^{-3}$ and $\sin \delta=0.1$ ), our benchmark is situated in the experimental $2 \sigma$ allowed region with $\Delta a_{\mu}=$ $2.24 \times 10^{-9}$. For its values, the $H$ contribution $\Delta a_{\mu}^{H}$ is significantly larger, with the $h^{\prime}$ contribution being $16.6 \%$ of the total.

\section{CONSTRAINTS ON THE MODEL}

This section is devoted to a discussion of constraints that the proposed scenario must satisfy, given the couplings of the extended scalar sector to fermions. We note here that in general, the off-diagonal couplings of the additional scalars in our model to down/up-type quarks are free parameters and can be very tiny, which is a relevant point that helps us stay safe from several existing bounds, as brought out below.

A second relevant point in the discussion below is that we assume that the predominant decay mode for the lightest state among the dark neutrinos $N_{i}$-i.e., $N_{1}$-is to lighter dark sector particles.

\section{A. Constraints from CHARM II and MINER $\nu \mathrm{A}$ :}

As discussed in Ref. [51], these experiments [86,87] constrain models attempting to explain the MB LEE and LSND based on results of high-energy $\nu-e$ scattering. A dark photon $\left(Z^{\prime}\right)$ model such as that discussed in Ref. [40] is tightly constrained for its chosen benchmark values, as shown in Ref. [51]. We also see that it is possible to evade this constraint provided the value of $\left|U_{\mu 4}\right|$ (the mixing between the muon and the up-scattered sterile neutrino in the proposed model of Ref. [40]) stays equal to or below $10^{-4}$. In order to check that our model is safe from these constraints, we calculate the cross section contribution from our process (with $H, h^{\prime}$ as mediators) for CHARM II and MINER $\nu \mathrm{A}$ and compare its value with that for the model in Ref. [40], with $\left|U_{\mu 4}\right|$ reduced to the safe value of $10^{-4}$. We find that the coherent cross section for our interaction stays more than an order of magnitude below this safe value, comfortably evading this constraint. We note that this is generically true for other recent models with scalar mediators, as also pointed out in Refs. [46,47].

We also note that elastic NC scattering of electrons with $H, h^{\prime}$ as mediators is not a concern, since the final state contains a $N_{2}$ which promptly decays to an $h^{\prime} N_{1}$ and subsequently a prompt $e^{+} e^{-}$. This does not observationally resemble $\mathrm{SM} \nu-e$ scattering.

\section{B. Constraints from T2K ND280:}

As discussed in Ref. [53], the T2K near-detector, ND280 [88], is in a position to provide bounds on new physics related to the MB LEE. Relevant to our work here, the specific decay $h^{\prime} \rightarrow e^{+} e^{-}$could be observable in this detector. Pair production can occur in the fine-grained detectors (FGD), in particular. We have calculated the number of events for our process and find 9 events in FGD1, using a momentum cutoff of $300 \mathrm{MeV}$ and an overall efficiency of $30 \%$. This is comfortably below their bounds.

In principle, at $\mathrm{T} 2 \mathrm{~K}$, such events may occur in the TPC also. In our model, however, this decay is prompt-hence, for detection in the TPC, the argon gas in it must act as both target and detection medium. Since the target mass is only $16 \mathrm{~kg}$, however, the number of events is unobservably small in our case. We also note that the threshold for detection in the TPC is around $200 \mathrm{MeV}$.

\section{Contributions to $\mathrm{NC} \nu$-nucleon scattering at high energies:}

Since the $H$ and $h^{\prime}$ in our model couple to neutrinos and quarks, a possible constraint arises from NC deep inelastic scattering (DIS) of neutrinos on nucleons, to which these scalars would contribute as mediators. At high energies, IceCube and DeepCore are a possible laboratory for new particles which are produced via such scattering [89,90]. In the process shown in Fig. 1, the decay time of $N_{2}$ (leading to an $e^{+} e^{-}$pair) is short enough to escape detection at these detectors. The distances traveled even at very high energies are much smaller than the resolution necessary to signal a double bang event: $\sim 1 \mathrm{~m}$ in DeepCore, and $\sim$ a few hundred meters in IceCube. In addition, we have checked that the high-energy NC cross section stays several orders of magnitude below the SM cross section. We also note that $N_{1}$ in our model is assumed to decay predominantly to invisible particles, again escaping detection in large detectors.

\section{Kaon and $B$-meson decay constraints:}

Prior to discussing specific cases, as a general remark, we note that in any heavy meson decay that involves $u, d$ quarks, one can radiate an $h^{\prime}$ which would promptly decay to an $e^{+} e^{-}$pair via the diagonal couplings between it and the quarks. While off-diagonal flavor-changing couplings in our model are arbitrarily small, the first-generation diagonal quark couplings to the scalars in our model are fixed by the requirements of fitting the LSND and MB data, and are approximately $\mathcal{O}\left(10^{-5}\right)$. These are small enough to suppress such decays by a factor $\mathcal{O}\left(10^{-10}\right)$, rendering them safe from existing upper bounds.

(1) $\mathrm{The} \operatorname{BR}\left(K_{L} \rightarrow \pi^{0} e^{+} e^{-}\right)<2.8 \times 10^{-10}$ at a $90 \%$ C.L. has been measured at $\mathrm{KTeV}$ [91]. Hence, in principle, the width for $K_{L} \rightarrow \pi^{0} h^{\prime}$ would contribute to this, while $K_{L} \rightarrow \pi^{0} H$ will not contribute, being kinematically forbidden. However, $\mathrm{KTeV}$ applies an invariant mass cut of $140 \mathrm{MeV}$ for the $e^{+} e^{-}$pair, making the bound inapplicable due to kinematics. We note also, as mentioned already, off-diagonal 
couplings of $h^{\prime}$ to $d, s$ quarks in our scenario are tiny. Also, the $\operatorname{BR}\left(h^{\prime} \rightarrow \gamma \gamma\right)$ is negligible. Therefore, the constraints from $K$ decays-e.g., $K_{L, S} \rightarrow \pi^{0} \gamma \gamma$ [92] - is not applicable.

(2) The E949 Collaboration [93] and NA62 Collaboration [94] have measured the process $K^{+} \rightarrow \pi^{+} \bar{\nu} \nu$, which could be mimicked by the $K^{+} \rightarrow \pi^{+} h^{\prime}$ decay in our scenario. Since $h^{\prime}$ decays primarily to $e^{+} e^{-}$, this means that it must be long-lived and escape the detector for this bound to apply. From Ref. [68], we see that given $h^{\prime}$ mass of $17 \mathrm{MeV}$ in our model, as long as lifetime is less than approximately $10^{-10} \mathrm{~s}$, one is safe from the constraint from invisibles. (In our model, $h^{\prime}$ has a lifetime $\simeq 1.8 \times 10^{-12} \mathrm{~s}$.) Moreover, as mentioned above, off-diagonal couplings of $h^{\prime}$ to $d, s$ quarks in our scenario are tiny.

(3) A light scalar coupled to muons can be emitted in the decay $K^{+} \rightarrow \mu^{+} \nu \phi$. This is constrained by the NA62 [95], as discussed in Ref. [64]. $H$ will evade these constraints because of its large mass, while $h^{\prime}$ lies outside the constrained range due to its short lifetime and small coupling to muons. In addition, we note that data collected by the NA48/2 experiment [96] can in principle provide constraints via observation of $K^{+} \rightarrow \mu^{+} \nu e^{+} e^{-}$, as noted in Ref. [64]. This analysis has recently been done [96], but with an invariant minimum-mass cut of $140 \mathrm{MeV}$ for the $e^{+} e^{-}$pair, which makes it inapplicable to $h^{\prime}$.

(4) The CHARM experiment $[97,98]$ has measured the displaced decay of neutral particles into $\gamma \gamma, e^{+} e^{-}$, and $\mu^{+} \mu^{-}$. The relevant decays are $K_{L} \rightarrow \pi^{0} h^{\prime}$ and $K^{+} \rightarrow \pi^{+} h^{\prime}$. Thus, in our model, $h^{\prime}$ can in principle be constrained by this experiment, but as discussed in Ref. [68], for $m_{h^{\prime}} \simeq 17 \mathrm{MeV}$, the lifetime in our case is much shorter than $10^{-10}$, which is the upper value set by this bound. Additionally, it is possible that CHARM, being sensitive to heavy neutral leptons given its dimensions, could have sensitivity to visible decays of $N_{1}$. As noted earlier, however, $N_{1}$ decays primarily to invisible states.

(5) The $K_{\mu 2}$ experiment [99] has measured the $K^{+} \rightarrow$ $\pi^{+} \phi$ process. For our benchmark point (Table I), $\operatorname{BR}\left(K^{+} \rightarrow \pi^{+} h^{\prime}\right) \simeq 4.2 \times 10^{-12}$ [100], which is very small compared with the upper limit $\sim 10^{-8}$.

(6) Similarly, the decay $B \rightarrow K^{*} e^{+} e^{-}$has been measured at the $\operatorname{LHCb}$ [101], $\operatorname{BR}\left(B \rightarrow K^{*} e^{+} e^{-}\right)=$ $(4.2 \pm 0.7) \times 10^{-7}$. In our model, this would correspond to $B \rightarrow K^{*} h^{\prime} / H$, with the latter going to $e^{+} e^{-}$. Given the $b \rightarrow s$ transition involved, and that couplings of $H, h^{\prime}$ to $b, s$ quarks can be arbitrarily small, we evade this constraint. Also, due to $2 m_{e}<m_{H}<$ $2 m_{\tau}$, the decays $B \rightarrow K^{(*)} H \rightarrow K^{(*)} \mu^{+} \mu^{-}$are subjected to strong constraints from $B \rightarrow K^{(*)} \mu^{+} \mu^{-}$at the $\mathrm{LHCb}$ [102]. However, in our model, we evade these constraints because of the smallness of $H$ coupling to $b, s$ quarks. Finally, in our model, it is worth mentioning that the branching ratios of $B \rightarrow$ $K^{(*)} \gamma \gamma / \nu \bar{\nu}$ are negligible.

\section{E. Constraints from neutrino trident production:}

The neutrino trident process [103] provides a sensitive probe of BSM physics at neutrino detectors, and has been measured [104-106]. It is relevant to our model given the couplings of the $H, h^{\prime}$ to muons, which are used for our explanation to the muon $g-2$ anomaly. Using the SM cross section and simple scaling, we have checked that our model is safe from this constraint.

\section{F. Pion decay constraints:}

$H, h^{\prime}$ couple to quarks, hence $H, h^{\prime}$ can mediate $\pi^{0}$ decay to $e^{+} e^{-}$. In the SM, this decay is loop-suppressed and consequently small. However, given the small couplings of the two scalars to the $u, d$ quarks $\left(\sim 10^{-5}\right)$ and the electron $\left(\sim 10^{-4}\right)$, we find that we are safely below this constraint.

\section{G. Collider bounds:}

At hadron colliders, the process $Z \rightarrow 4 \ell$ proceeds via $q \bar{q} \rightarrow Z^{*} \rightarrow \bar{\ell} \ell$, along with a $\gamma^{*}$ attached to one of the external legs (either the quarks or leptons) and with the creation of a lepton pair from the $\gamma^{*}$. This process has been measured at the LHC [107]. The $Z$ and $\gamma$ in principle can be replaced by $H, h^{\prime}$. The bound from LHC, however, applies an $\ell^{+} \ell^{-}$invariant mass cut of $4 \mathrm{GeV}$, and hence does not apply to our situation.

Since $H$ couples to leptons and quarks, $H$ can be radiated from these in external and internal legs in any process, and then $H \rightarrow h^{\prime} h^{\prime}$ is possible, leading to two collimated pairs of $e^{+} e^{-}$, which will look like two leptons. An LHC search was conducted [108], and no significant deviation or excess was found. As discussed in Ref. [68], given the fact that the $H$ to lepton/quark coupling is small $\left(\lesssim 10^{-4}\right)$, and also that the $H \rightarrow h^{\prime} h^{\prime}$ decay width is small due to the smallness of its coupling, the contribution will stay within the $1 \%$ level.

If $H, h^{\prime}$ couple to $b$ and $s$ quarks, then the decay $B_{s} \rightarrow \mu^{+} \mu^{-}$can be mediated by them. This decay has been measured by both the LHCb and CMS (see Refs. [109-113]). However, in our model the couplings to $b, s$ quarks can be arbitrarily small, hence this constraint can be avoided.

\section{H. Constraints on $y_{e}$ and $m_{h^{\prime}}$ from dark photon searches:}

A dark photon search looks for its decay to a lepton pair. These bounds can be translated [114,115] to constraints on a light scalar which couples to leptons. Specifically, translated constraints relevant to our scenario arise from KLOE [116] and BABAR [117]. Current values of $y_{e}$ and $m_{h^{\prime}}$ in our scenario are safe from these bounds, but they will be tested in the future by Belle-II [118]. 


\section{Constraints on $y_{e}$ and $m_{h^{\prime}}$ from electron beam dump experiments:}

A light scalar with couplings to electrons could be searched for $[63,64]$ in beam dump experiments via its decay to an $e^{+} e^{-}$pair or photons. Relevant to the mass range under consideration here are the experiments E137 [119], E141 [120], ORSAY [121], and NA64 [122]. While our present values are outside the forbidden regions, they will be tested in the future by the HPS fixed target experiment [123], which will scatter electrons on tungsten.

\section{J. Constraints on $y_{e}$ and $m_{H}$ from dark photon searches:}

KLOE [124] searched for $e^{+} e^{-} \rightarrow U \gamma$, followed by $U$ decays to $\pi^{+} \pi^{-} / \mu^{+} \mu^{-}$, leading to the constraint on $y_{e}(\sim 2 \times$ $\left.10^{-4}\right)$ at $m_{U}=750 \mathrm{MeV}$. In our scenario, replacing $U$ by $H$, we note that the production of $\pi^{+} \pi^{-} / \mu^{+} \mu^{-}$by it in KLOE will be very suppressed due to its tiny coupling to $u, d$ quarks and its predominant semivisible decay $\left(H \rightarrow e^{+} e^{-}+\not E\right)$. Moreover, both visible and invisible final-states searches by $B A B A R[117,125]$ put upper limits on $y_{e}$ at $m_{H}$, which can be evaded by $H$ due to its predominant semivisible decay.

\section{K. Constraints on $\boldsymbol{y}_{\boldsymbol{\mu}}$ and $\boldsymbol{m}_{\boldsymbol{H}}$ from colliders:}

$B A B A R$ has provided constraints $[64,118]$ on these parameters via their search for $e^{+} e^{-} \rightarrow \mu^{+} \mu^{-} \phi$, where $\phi$ is a generic light scalar. Our values, while currently in conformity with these bounds, will be tested in the future by Belle-II [118]. In addition, BABAR [126] constrains a dark leptophillic light scalar with couplings which are proportional to $m_{f} / v$. This set of constraints does not apply in our case, since our couplings for $h^{\prime}$ and $H$ do not have this proportionality.

\section{Contribution from the new scalars to the electron $g-2$ anomaly:}

The (positive) one-loop contribution in our model allows us to explain the observed value of $\Delta a_{\mu}$. A similar (positive) contribution is made to $\Delta a_{e}$ by both $h^{\prime}$ and $H$, which we have computed, summed, and found to be $\Delta a_{e}=4 \times 10^{-14}$. This is well within the present uncertainties in this quantity. We note that our model allows the possibility of having negative off-diagonal Yukawa couplings to $\Delta a_{e}$ by both $h^{\prime}$ and $H$. This affords flexibility in varying this contribution and keeping it within acceptable limits, as well as possibly explaining the current $\Delta a_{e}$ discrepancy at the one-loop level, as discussed in Ref. [47].

\section{CONCLUSIONS}

Evidence for anomalous signals at low-energy noncollider experiments in general, and short-baseline neutrino experiments in particular, has been gradually increasing over time, and has firmed up significantly over the past decade or so. Specifically, with reference to the LSND and MB excesses, it has gradually become evident that one may choose several different approaches towards understanding their origin, and these choices can lead down divergent and nonoverlapping paths. ${ }^{4}$

An important premise underlying our effort in this paper is that a common, nonoscillation, new physics explanation exists for both LSND and MB. Furthermore, our effort is guided by the belief that such an explanation could not only yield a long-sought extension to the SM, but also delineate the contours of the portal connecting the SM to the dark sector, as well as shed some light on other related but as yet unresolved questions.

Pursuant to this, in the scenario presented here, the extension to the SM that these experiments lead to comprises the well-known 2HDM. Access to the dark sector is achieved via mass mixing with a (dark) relatively light singlet scalar and via the presence of heavier dark neutrinos in allowed gauge-invariant terms in the Lagrangian. Two of the three $C P$-even scalars in the model are relatively light $\left(m_{h^{\prime}} \simeq 17 \mathrm{MeV}\right.$ and $\left.m_{H} \simeq 750 \mathrm{MeV}\right)$ and participate in the interaction that generates the excesses in LSND and MB, as well as contribute to the value of the muon $g-2$. Similarly, two of the three dark neutrinos not only participate in important ways in the interaction ${ }^{5}$ in LSND and MB, but also, along with the third neutrino, generate neutrino masses via a simple seesaw mechanism.

The sub-GeV scalars in our model can be searched for in a variety of experiments. The masses of $h^{\prime}$ and $H$ lie especially close to existing bounds from electron beamdump experiments like E141 [120] and BABAR [117], respectively. Thus, $H$ can be searched for in Belle-II [118] and $h^{\prime}$ in HPS [123]. The dark fermions in our model are amenable to searches in several upcoming experimentse.g., DUNE ND [127] (for a more detailed discussion and references, see Refs. [128,129]).

In the near future, the MicroBooNE experiment [130-132] will provide first indications of whether the low-energy electronlike event excesses in MB and LSND are due to electrons or photons. In the scenario presented here, the $h^{\prime}$ has a very short lifetime prior to decay to an $e^{+} e^{-}$pair. At the energies under consideration, it would travel about 5-12 $\mathrm{mm}$ in the detector. Since tracks with a gap of greater than $1 \mathrm{~cm}$ would be interpreted as photons, most events resulting from our scenario would look like an excess of electrons in MicroBooNE, while the high-energy ones could be mistaken for photons with short gaps.

\footnotetext{
${ }^{4}$ For example, one could conclude that these anomalies are due to poorly understood SM backgrounds or detector-specific systematic effects, as opposed to trying to understand them via active-sterile neutrino oscillations. Clearly, these choices would subsequently entail very different theoretical and experimental efforts towards their eventual resolution.

${ }^{5}$ The third dark neutrino, $N_{3}$, with $m_{N_{3}} \simeq 10 \mathrm{GeV}$, does not participate because of kinematics, not dynamics.
} 
A $d E / d x$ analysis would be required to actually detect that the events are $e^{+} e^{-}$pairs rather than electrons, which should also be possible with more data.

With respect to the scalar search for the $h^{\prime}$, we mention two existing experimental hints which are interesting: $a$ ) a significant excess in the $10-20 \mathrm{MeV}$ invariant mass bin of electronlike FGD1-TPC pairs detected by the T2K ND280 detector, (see Fig. 11 in Ref. [88]), and $b$ ) the higherthan-expected central value for the width $\Gamma\left(\pi^{0} \rightarrow e^{+} e^{-}\right)$ observed by the $\mathrm{KTeV}$ experiment [133], signifying the possible existence of a scalar with mass $\simeq 17 \mathrm{MeV}$. Additionally, we would like to point out that the Kaon DAR search, planned at the JSNS ${ }^{2}$ experiment $[134,135]$, is in a position to provide a test of the proposal presented in this work via its flux of high-energy $\nu_{\mu}$ [136].

In conclusion, we are hopeful that the long-standing and statistically significant anomalous results of LSND and $\mathrm{MB}$, along with the connection established between them via the simple model presented here will help motivate a more focused search for these particles in ongoing and future experiments.

\section{ACKNOWLEDGMENTS}

R. G. would like to express his sincere appreciation to William Louis for his help with our many questions on MB and LSND. He is thankful to Boris Kayser and Geralyn Zeller for helpful discussions, and acknowledges useful email exchanges with Lukas Koch, Teppei Katori, and Zoya Vallari. He is grateful to the Theory Division and the Neutrino Physics Center at Fermilab for hospitality and visits where this work benefitted from discussions and a conducive environment. S. R. is grateful to Fermilab for support via the Rajendran Raja Fellowship. W. A., R. G., and S.R. also acknowledge support from the XII Plan Neutrino Project of the Department of Atomic Energy and the High Performance Cluster Facility at HRI (http://www .hri.res.in/cluster/).
[1] M. Tanabashi et al. (Particle Data Group), Phys. Rev. D 98, 030001 (2018).

[2] K. Arun, S. B. Gudennavar, and C. Sivaram, Adv. Space Res. 60, 166 (2017).

[3] F. Kahlhoefer, Int. J. Mod. Phys. A 32, 1730006 (2017).

[4] J. M. Gaskins, Contemp. Phys. 57, 496 (2016).

[5] G. Bertone, D. Hooper, and J. Silk, Phys. Rep. 405, 279 (2005).

[6] J. L. Feng, Annu. Rev. Astron. Astrophys. 48, 495 (2010).

[7] S. Pascoli and J. Turner, Nature (London) 580, 323 (2020).

[8] L. Canetti, M. Drewes, and M. Shaposhnikov, New J. Phys. 14, 095012 (2012).

[9] Q. R. Ahmad et al. (SNO Collaboration), Phys. Rev. Lett. 87, 071301 (2001).

[10] Y. Fukuda et al. (Super-Kamiokande Collaboration), Phys. Rev. Lett. 81, 1562 (1998).

[11] K. Abe, N. Abgrall, H. Aihara, T. Akiri, J. B. Albert, C. Andreopoulos, S. Aoki, A. Ariga, T. Ariga, S. Assylbekov et al. (T2K Collaboration), Phys. Rev. D 88, 032002 (2013).

[12] J. K. Ahn et al. (RENO Collaboration), Phys. Rev. Lett. 108, 191802 (2012).

[13] M. Maltoni, https://doi.org/10.5281/zenodo.1287015 (2018).

[14] J. P. Miller, E. de Rafael, and B. Roberts, Rep. Prog. Phys. 70, 795 (2007).

[15] R. H. Parker, C. Yu, W. Zhong, B. Estey, and H. Mller, Science 360, 191 (2018).

[16] J. Ahn et al. (KOTO Collaboration), Phys. Rev. Lett. 122, 021802 (2019).

[17] D. London, arXiv:1911.06238.

[18] L. Delle Rose, S. Khalil, S. J. King, and S. Moretti, Front. Phys. 7, 73 (2019).
[19] A. Aguilar-Arevalo et al. (LSND Collaboration), Phys. Rev. D 64, 112007 (2001).

[20] A. Aguilar-Arevalo et al. (MiniBooNE Collaboration), Phys. Rev. D 103, 052002 (2021).

[21] G. Bennett et al. (Muon g-2 Collaboration), Phys. Rev. D 73, 072003 (2006).

[22] M. Lindner, M. Platscher, and F. S. Queiroz, Phys. Rep. 731, 1 (2018).

[23] K. Eitel, Prog. Part. Nucl. Phys. 48, 89 (2002).

[24] A. A. Aguilar-Arevalo et al. (MiniBooNE Collaboration), Phys. Rev. Lett. 121, 221801 (2018).

[25] K. N. Abazajian et al., arXiv:1204.5379.

[26] G. H. Collin, C. A. Argüelles, J. M. Conrad, and M. H. Shaevitz, Nucl. Phys. B908, 354 (2016).

[27] G. H. Collin, C. A. Argüelles, J. M. Conrad, and M. H. Shaevitz, Phys. Rev. Lett. 117, 221801 (2016).

[28] J. M. Conrad and M. H. Shaevitz, Adv. Ser. Dir. High Energy Phys. 28, 391 (2018).

[29] S. Gariazzo, C. Giunti, M. Laveder, and Y. F. Li, J. High Energy Phys. 06 (2017) 135.

[30] M. Dentler, A. Hernández-Cabezudo, J. Kopp, P. A. Machado, M. Maltoni, I. Martinez-Soler, and T. Schwetz, J. High Energy Phys. 08 (2018) 010.

[31] A. Diaz, C. A. Argüelles, G. H. Collin, J. M. Conrad, and M. H. Shaevitz, Phys. Rep. 884, 1 (2020).

[32] S. Palomares-Ruiz, S. Pascoli, and T. Schwetz, J. High Energy Phys. 09 (2005) 048.

[33] S. N. Gninenko, Phys. Rev. Lett. 103, 241802 (2009).

[34] S. N. Gninenko, Phys. Rev. D 83, 015015 (2011).

[35] M. Masip, P. Masjuan, and D. Meloni, J. High Energy Phys. 01 (2013) 106.

[36] Y. Bai, R. Lu, S. Lu, J. Salvado, and B. A. Stefanek, Phys. Rev. D 93, 073004 (2016). 
[37] J. Liao and D. Marfatia, Phys. Rev. Lett. 117, 071802 (2016).

[38] M. Carena, Y.-Y. Li, C. S. Machado, P. A. N. Machado, and C. E. M. Wagner, Phys. Rev. D 96, 095014 (2017).

[39] J. Asaadi, E. Church, R. Guenette, B. J. P. Jones, and A. M. Szelc, Phys. Rev. D 97, 075021 (2018).

[40] E. Bertuzzo, S. Jana, P. A. N. Machado, and R. Zukanovich Funchal, Phys. Rev. Lett. 121, 241801 (2018).

[41] P. Ballett, S. Pascoli, and M. Ross-Lonergan, Phys. Rev. D 99, 071701 (2019).

[42] A. Ioannisian, arXiv:1909.08571.

[43] O. Fischer, A. Hernández-Cabezudo, and T. Schwetz, Phys. Rev. D 101, 075045 (2020).

[44] M. Dentler, I. Esteban, J. Kopp, and P. Machado, Phys. Rev. D 101, 115013 (2020).

[45] A. de Gouvêa, O. L. G. Peres, S. Prakash, and G. V. Stenico, J. High Energy Phys. 07 (2020) 141.

[46] A. Datta, S. Kamali, and D. Marfatia, Phys. Lett. B 807, 135579 (2020).

[47] B. Dutta, S. Ghosh, and T. Li, Phys. Rev. D 102, 055017 (2020).

[48] W. Abdallah, R. Gandhi, and S. Roy, J. High Energy Phys. 12 (2020) 188.

[49] A. Abdullahi, M. Hostert, and S. Pascoli, Phys. Lett. B 820, 136531 (2021).

[50] A. A. Aguilar-Arevalo et al. (MiniBooNE DM Collaboration), Phys. Rev. D 98, 112004 (2018).

[51] C. A. Argüelles, M. Hostert, and Y.-D. Tsai, Phys. Rev. Lett. 123, 261801 (2019).

[52] J. R. Jordan, Y. Kahn, G. Krnjaic, M. Moschella, and J. Spitz, Phys. Rev. Lett. 122, 081801 (2019).

[53] V. Brdar, O. Fischer, and A. Y. Smirnov, Phys. Rev. D 103, 075008 (2021).

[54] P. A. N. Machado, https://doi.org/10.5281/zenodo.3959609 (2020).

[55] H. Brown et al. (Muon g-2 Collaboration), Phys. Rev. Lett. 86, 2227 (2001).

[56] F. Jegerlehner and A. Nyffeler, Phys. Rep. 477, 1 (2009).

[57] J. L. Holzbauer, J. Phys. Conf. Ser. 770, 012038 (2016).

[58] T. Kinoshita and W. J. Marciano, Theory of the Muon Anomalous Magnetic Moment (World Scientific, 1990), Vol. 7, pp. 419-478, https://doi.org/10.1142/ 9789814503273_0010.

[59] Y.-F. Zhou and Y.-L. Wu, Eur. Phys. J. C 27, 577 (2003).

[60] V. Barger, C.-W. Chiang, W.-Y. Keung, and D. Marfatia, Phys. Rev. Lett. 106, 153001 (2011).

[61] D. Tucker-Smith and I. Yavin, Phys. Rev. D 83, 101702 (2011).

[62] C.-Y. Chen, H. Davoudiasl, W. J. Marciano, and C. Zhang, Phys. Rev. D 93, 035006 (2016).

[63] Y.-S. Liu, D. McKeen, and G. A. Miller, Phys. Rev. Lett. 117, 101801 (2016).

[64] B. Batell, N. Lange, D. McKeen, M. Pospelov, and A. Ritz, Phys. Rev. D 95, 075003 (2017).

[65] W. Marciano, A. Masiero, P. Paradisi, and M. Passera, Phys. Rev. D 94, 115033 (2016).

[66] L. Wang, J. M. Yang, and Y. Zhang, Nucl. Phys. B924, 47 (2017).

[67] J. Liu, C. E. Wagner, and X.-P. Wang, J. High Energy Phys. 03 (2019) 008.
[68] J. Liu, N. McGinnis, C. E. Wagner, and X.-P. Wang, J. High Energy Phys. 04 (2020) 197.

[69] S. Jana, P. K. Vishnu, and S. Saad, Phys. Rev. D 101, 115037 (2020).

[70] T. Lee, Phys. Rev. D 8, 1226 (1973).

[71] G. Branco, P. Ferreira, L. Lavoura, M. Rebelo, M. Sher, and J. P. Silva, Phys. Rep. 516, 1 (2012).

[72] G. C. Branco, L. Lavoura, and J. P. Silva, CP Violation, International Series of Monographs on Physics (Oxford University Press, UK, 1999), Vol. 103.

[73] S. Davidson and H. E. Haber, Phys. Rev. D 72, 035004 (2005); 72, 099902(E) (2005).

[74] P. de Salas, D. Forero, C. Ternes, M. Tortola, and J. Valle, Phys. Lett. B 782, 633 (2018).

[75] P. Junnarkar and A. Walker-Loud, Phys. Rev. D 87, 114510 (2013).

[76] A. Crivellin, M. Hoferichter, and M. Procura, Phys. Rev. D 89, 054021 (2014).

[77] M. Hoferichter, J. Ruiz de Elvira, B. Kubis, and U.-G. Meißner, Phys. Rev. Lett. 115, 092301 (2015).

[78] R. J. Hill, Phys. Rev. D 81, 013008 (2010).

[79] D. Z. Freedman, Phys. Rev. D 9, 1389 (1974).

[80] A. A. Aguilar-Arevalo et al. (MiniBooNE Collaboration), Phys. Rev. Lett. 121, 221801 (2018).

[81] C. Athanassopoulos et al. (LSND Collaboration), Phys. Rev. C 58, 2489 (1998).

[82] G. S. Karagiorgi, Ph.D. thesis, 2010, https://www.osti.gov/ biblio/1000269-1F5G7k/.

[83] W. Louis (private communication).

[84] R. Jackiw and S. Weinberg, Phys. Rev. D 5, 2396 (1972).

[85] J. P. Leveille, Nucl. Phys. B137, 63 (1978).

[86] P. Vilain, G. Wilquet, R. Beyer, W. Flegel, H. Grote, T. Mouthuy, H. veras, J. Panman, A. Rozanov, K. Winter et al., Phys. Lett. B 335, 246 (1994).

[87] E. Valencia et al. (MINERvA Collaboration), Phys. Rev. D 100, 092001 (2019).

[88] K. Abe et al. (T2K Collaboration), J. High Energy Phys. 10 (2020) 114.

[89] P. Coloma, P. A. Machado, I. Martinez-Soler, and I. M. Shoemaker, Phys. Rev. Lett. 119, 201804 (2017).

[90] P. Coloma, Eur. Phys. J. C 79, 748 (2019).

[91] A. Alavi-Harati et al. (KTeV Collaboration), Phys. Rev. Lett. 93, 021805 (2004).

[92] E. Abouzaid et al. (KTeV Collaboration), Phys. Rev. D 77, 112004 (2008).

[93] A. Artamonov et al. (BNL-E949 Collaboration), Phys. Rev. D 79, 092004 (2009).

[94] G. Ruggiero, J. Phys. Conf. Ser. 1526, 012003 (2020).

[95] S. Martellotti, arXiv:1510.00172.

[96] D. Madigozhin (NA48/2 Collaboration), EPJ Web Conf. 206, 05001 (2019).

[97] F. Bergsma et al. (CHARM Collaboration), Phys. Lett. 128B, 361 (1983).

[98] F. Bergsma et al. (CHARM Collaboration), Phys. Lett. 166B, 473 (1986).

[99] T. Yamazaki et al., Phys. Rev. Lett. 52, 1089 (1984).

[100] B. Batell, A. Freitas, A. Ismail, and D. Mckeen, Phys. Rev. D 100, 095020 (2019).

[101] R. Aaij et al. (LHCb Collaboration), J. High Energy Phys. 05 (2013) 159. 
[102] R. Aaij et al. (LHCb Collaboration), Phys. Rev. Lett. 115, 161802 (2015).

[103] S.-F. Ge, M. Lindner, and W. Rodejohann, Phys. Lett. B 772, 164 (2017).

[104] D. Geiregat et al. (CHARM-II Collaboration), Phys. Lett. B 245, 271 (1990).

[105] S. Mishra et al. (CCFR Collaboration), Phys. Rev. Lett. 66, 3117 (1991).

[106] T. Adams et al. (NuTeV Collaboration), in 29th International Conference on High-Energy Physics (1998), pp. 631-634 [arXiv:hep-ex/9811012].

[107] J. L. Rainbolt and M. Schmitt, Phys. Rev. D 99, 013004 (2019).

[108] G. Aad et al. (ATLAS Collaboration), J. High Energy Phys. 02 (2016) 062.

[109] R. Aaij et al. (LHCb Collaboration), Phys. Rev. Lett. 111, 101805 (2013).

[110] S. Chatrchyan et al. (CMS Collaboration), Phys. Rev. Lett. 111, 101804 (2013).

[111] V. Khachatryan et al. (CMS, LHCb Collaborations), Nature (London) 522, 68 (2015).

[112] R. Aaij et al. (LHCb Collaboration), Phys. Rev. Lett. 118, 191801 (2017).

[113] M. Aaboud et al. (ATLAS Collaboration), J. High Energy Phys. 04 (2019) 098.

[114] D. S. M. Alves and N. Weiner, J. High Energy Phys. 07 (2018) 092.

[115] S. Knapen, T. Lin, and K. M. Zurek, Phys. Rev. D 96, 115021 (2017).

[116] A. Anastasi et al., Phys. Lett. B 750, 633 (2015).

[117] J. Lees et al. (BABAR Collaboration), Phys. Rev. Lett. 113, 201801 (2014).

[118] B. Batell, A. Freitas, A. Ismail, and D. Mckeen, Phys. Rev. D 98, 055026 (2018).
[119] J. Bjorken, S. Ecklund, W. Nelson, A. Abashian, C. Church, B. Lu, L. Mo, T. Nunamaker, and P. Rassmann, Phys. Rev. D 38, 3375 (1988).

[120] E. Riordan et al., Phys. Rev. Lett. 59, 755 (1987).

[121] M. Davier and H. Nguyen Ngoc, Phys. Lett. B 229, 150 (1989).

[122] D. Banerjee et al. (NA64 Collaboration), Phys. Rev. Lett. 120, 231802 (2018).

[123] M. Battaglieri et al., Nucl. Instrum. Methods Phys. Res., Sect. A 777, 91 (2015).

[124] A. Anastasi et al. (KLOE-2 Collaboration), Phys. Lett. B 784, 336 (2018).

[125] J. Lees et al. (BABAR Collaboration), Phys. Rev. Lett. 119, 131804 (2017).

[126] J. P. Lees et al. (BABAR Collaboration), Phys. Rev. Lett. 125, 181801 (2020).

[127] C. Adams et al. (LBNE Collaboration), arXiv:1307.7335.

[128] P. Ballett, T. Boschi, and S. Pascoli, J. High Energy Phys. 03 (2020) 111.

[129] J. M. Berryman, A. de Gouvea, P. J. Fox, B. J. Kayser, K. J. Kelly, and J. L. Raaf, J. High Energy Phys. 02 (2020) 174.

[130] D. Caratelli (MicroBooNE Collaboration), Proc. Sci., ICHEP2020 (2021) 118 [arXiv:2012.08085].

[131] N. Foppiani (MicroBooNE Collaboration), arXiv:1905 .05325 .

[132] D. Kaleko, Ph.D. thesis, Columbia University, 2017, https://doi.org/10.7916/D87D3260.

[133] E. Abouzaid et al. (KTeV Collaboration), Phys. Rev. D 75, 012004 (2007).

[134] S. Ajimura et al., arXiv:1705.08629.

[135] J. Jordan, https://doi.org/10.5281/zenodo.4122990 (2020).

[136] We thank William Louis for drawing our attention to this possibility. 